\title{
Pathway analysis of a genome-wide association study on a long non-coding RNA expression profile in oral squamous cell carcinoma
}

\author{
YONG-LE QIU ${ }^{1}$, YUAN-HANG LIU ${ }^{2}$, JIAN-DONG BAN $^{3}$, WEN-JING WANG ${ }^{1}$, \\ MEI HAN $^{4}$, PENG KONG ${ }^{4}$ and BING-HUI LI ${ }^{1}$ \\ ${ }^{1}$ Department of Surgery, Fourth Affiliated Hospital, Hebei Medical University, Shijiazhuang, Hebei 050017; \\ ${ }^{2}$ Department of Stomatology, Second Hospital of Shijiazhuang, Shijiazhuang, Hebei 050000; \\ ${ }^{3}$ Department of Stomatology, Hebei Eye Hospital, Xingtai, Hebei 054000; \\ ${ }^{4}$ Department of Biochemistry and Molecular Biology, Hebei Medical University, \\ Shijiazhuang, Hebei 050000, P.R. China
}

Received June 8, 2018; Accepted November 1, 2018

DOI: $10.3892 /$ or.2018.6870

\begin{abstract}
Long non-coding RNAs (lncRNAs) have been consistently demonstrated to be involved in oral squamous cell carcinoma (OSCC) as either tumor oncogenes or tumor suppressors. However, the underlying mechanisms of OSCC tumorigenesis and development have not yet been fully elucidated. The expression profiles of mRNAs and lncRNAs in OSCC were analyzed by a microarray assay. To verify the results of the microarray, 10 differentially expressed lncRNAs were randomly selected and measured by quantitative RT-PCR (qRT-PCR). Gene Ontology (GO) and metabolic pathway analyses were performed to analyze gene function and identify enriched pathways. Subsequently, two independent algorithms were used to predict the target genes of the lncRNAs. We identified 2,294 lncRNAs and 1,938 mRNAs that were differentially expressed in all three OSCC tissues by a microarray assay. Through the construction of co-expression networks of differentially expressed genes, 4 critical lncRNAs nodes were identified as potential key factors in the pathogenesis of OSCC. Expression of the 4 critical lncRNA nodes was not associated with age, sex, smoking or tumor location $(\mathrm{P}>0.05)$ but was positively correlated with clinical stage, lymphatic metastasis, distant metastasis and survival status $(\mathrm{P}<0.05)$. Kaplan-Meier analysis demonstrated that low expression levels of these 4 critical lncRNA nodes contributed to poor median progression-free survival (PFS) and overall survival (OS) $(\mathrm{P}<0.05)$. GO and pathway analyses indicated that the
\end{abstract}

Correspondence to: Dr Bing-Hui Li, Department of Surgery, Fourth Affiliated Hospital, Hebei Medical University, 12 Health Road, Shijiazhuang, Hebei 050017, P.R. China

E-mail: sss_qiu123@163.com

Key words: oral squamous cell carcinoma, long non-coding RNA, lncRNA, mRNA, microarray, pathway analysis functions and enriched pathways of many dysregulated genes are associated with cancer. Potential target genes of dysregulated lncRNAs were enriched in 43 metabolic pathways, with cancer pathways being the primary enrichment pathways. In summary, we analyzed the profile of IncRNAs in OSCC and identified the functions and enriched metabolic pathways of both dysregulated mRNAs and the target genes of dysregulated lncRNAs, providing new insights into molecular markers and therapeutic targets for OSCC.

\section{Introduction}

Oral squamous cell carcinoma (OSCC) is one of the most common malignancies in the head and neck region. OSCC is derived from oral mucosal epithelium and is characterized by its strong local infiltration and cervical lymph node metastasis. Many factors, including smoking, can lead to the tumorigenesis and development of OSCC, which has a poor prognosis and low overall survival rate $(1,2)$. OSCC not only affects the lives of late-stage patients but also affects their ability to chew and swallow and their appearance. Although surgery, radiotherapy and chemotherapy have made considerable progress in terms of treatment, the prognosis of OSCC patients remains poor. Early detection and radical treatment of tumors both present challenges and opportunities for cancer researchers (3). The solution to this issue depends on a comprehensive understanding of the molecular mechanisms of tumorigenesis and development. Many research groups are actively involved in the study of OSCC pathogenesis; however, the underlying mechanisms of OSCC tumorigenesis and development have not been fully elucidated (4-6). Therefore, further studies focusing on the mechanisms of OSCC need to be performed to improve early diagnosis, targeted therapy and prognosis.

Long non-coding RNAs (lncRNAs) are a group of non-protein coding RNAs $>200 \mathrm{nt}$ (7). It is estimated that only $2 \%$ of the human genome is transcribed into mRNAs, while $70-90 \%$ of the genome is transcribed into lncRNAs (7). lncRNAs play important roles in epigenetic modification, 
transcription and post-transcriptional regulation, maintenance of normal tissue development and differentiation $(3,4)$. Recently, increasing evidence has indicated that lncRNAs exert vital roles in a number of biological processes, including cell metabolism and immune response, through comprehensive mechanisms (8). IncRNAs that affect tumorigenesis and development are considered novel candidates for targeted tumor therapy (9). According to previous research, IncRNAs are closely correlated with tumorigenesis and development of esophageal, liver, lung and breast cancer (10-13). IncRNAs affect the expression of mRNA by regulating the transcription and stability of their target genes (14). Delineating the lncRNA-mRNA coexpression network is an important method for analyzing the functional and regulatory mechanisms of lncRNAs.

The aim of the present study was to identify dysregulated lncRNAs and mRNAs in OSCC patients. The results of the present study indicated that abnormal expression of lncRNAs may contribute to the tumorigenesis and development of OSCC. Furthermore, the present study provides new insight into the molecular markers and therapeutic targets for OSCC.

\section{Materials and methods}

Samples. Seventy-two oral squamous cell carcinoma (OSCC) tissues and paired adjacent normal tissues (excised $2 \mathrm{~cm}$ from the tumor-free margin) were obtained from the Fourth Affiliated Hospital of Hebei Medical University between January 2015 and October 2016 and were pathologically confirmed to be OSCC ( 32 were $<50$ and 40 were $\geq 50$ years; 35 male and 37 female patients). The present study was approved by the Ethics Committee of the Fourth Affiliated Hospital of Hebei Medical University (Shijiazhuang, China) and written informed consent was obtained from all subjects. None of the patients received radiotherapy, chemotherapy or other cancer treatment before tumor resection. Tumors were histologically graded according to the World Health Organization (WHO) standards. Classification of tumors was performed according to the TNM staging revised by the International Union Against Cancer (UICC) (https://www.uicc.org/).

All 72 patients were followed up by telephone and outpatient methods after discharge with assessments of their general condition and clinical symptoms and imaging examinations. The starting point of follow-up was the date of surgery or pathological biopsy, and the follow-up period ended on April 30, 2018. At the end of the follow-up period, 49 patients were still alive, and 23 had died; and no patient was lost to follow-up. Progression-free survival (PFS) was defined as the time between diagnosis and progression of the disease, and overall survival (OS) was defined as the time from diagnosis to death or last follow-up.

Microarray assay. Of these samples, three tissue pairs including tongue cancer ( $\mathrm{T} 1 \mathrm{~N} 2 \mathrm{M} 0)$, gingival carcinoma (T2N0M0) and carcinoma of the buccal mucosa (T3N1M0) were used for microarray analysis. The microarray (SBC human 4*180K lncRNA array; Shanghai Biotechnology Corp., Shanghai, China) used in the present study was capable of detecting 77,103 lncRNAs and 18,853 mRNAs and covered core databases, such as GENCODE v21 (https://www.gencodegenes.org), Lncipedia v3.1 (https://lncipedia.org), Ensembl (http://asia.ensembl.org) and Agilent_ncRNA (https://earray. chem.agilent.com).

Total RNA extraction. Total RNA was extracted from frozen samples by TRIzol (Invitrogen; Thermo Fisher Scientific, Inc., Waltham, MA, USA). Quantitation was preformed using a NanoDrop 2000 spectrophotometer (NanoDrop Technologies; Thermo Fisher Scientific, Inc.). Denaturing agarose gel electrophoresis was used to assess the integrity of total RNA extracted from tissues.

cDNA synthesis, labeling and hybridization. Qualified total RNA was used for the synthesis of cDNA followed by fluorescent labeling according to the manufacturer's instructions with the Agilent's Low Input Quick Amp WT Labeling kit (Agilent Technologies, Santa Clara, CA, USA). The labeled cRNA was purified with RNeasy Mini kit (Qiagen GmbH, Hilden, Germany) and hybridization was performed at $65^{\circ} \mathrm{C}$ for $17 \mathrm{~h}$.

Microarray data analysis. An Agilent Microarray Scanner (Agilent Technologies) was used in the present study. Data were obtained using Feature Extraction software 10.7 (Agilent Technologies). Raw data were normalized by Quantile algorithm, Gene Spring Software 11.0 (Agilent Technologies). The IncRNAs and mRNAs were considered to be differentially expressed when the fold change (FC) was $>2$ $(\mathrm{P}<0.05)$. A volcano plot was used to visualize differentially expressed genes and was subsequently processed for hierarchical clustering analysis using Gene Spring Software 11.0 (Agilent Technologies). Finally, Pearson correlation coefficients between differentially expressed lncRNAs and mRNAs were calculated, and co-expression networks of lncRNAs and mRNAs were constructed.

Gene Ontology (GO) and Kyoto Encyclopedia of Genes and Genomes (KEGG) pathway analysis. GO terms were used to annotate and classify gene function. The differentially expressed genes were put into the Database for Annotation, Visualization and Integrated Discovery (DAVID; http://david. abcc.ncifcrf.gov/) v6.8, which utilizes GO to identify the molecular function represented in the gene profile. Furthermore, we used KEGG to analyze the potential functions of these genes in metabolic pathways. $\mathrm{P}<0.05$ was recommended as a cut-off value.

Prediction of IncRNA target genes. Two independent algorithms were used to predict the target genes of dysregulated lncRNAs. The first algorithm was performed to predict cis-acting target genes using the University of California Santa Cruz (UCSC; Santa Cruz, CA, USA) genome browser (http://genome.ucsc.edu/). Genes transcribed within a 10-kb window upstream or downstream of IncRNAs were considered cis target genes. The second algorithm predicted trans-acting target genes using RNAplex 0.2 software (http://www.bioinf. uni-leipzig.de/Software/RNAplex) based on RNA duplex energy prediction and mRNA sequence complementation according to the previous reference (15).

$q R T-P C R$. Differentially expressed lncRNAs from microarray data were randomly selected for qRT-PCR. Reverse 
Table I. Primers used for qRT-PCR.

\begin{tabular}{lll}
\hline lncRNA & \multicolumn{1}{c}{ Forward primer (5'-3') } & \multicolumn{1}{c}{ Reverse primer $\left(5^{\prime}-3^{\prime}\right)$} \\
\hline lnc-MANSC4-8:1 & AAGGAAAACAACAGAAGAACAC & GCCAGCTTAAAGAGACAAATA \\
CXCR2P1 & AGGGGAGTATGGGGAGTGATG & GGGCCAAGGTGTTTTCTTTTTA \\
NRIR & CCAAGAAAAGAGGGCTTAAAATGAA & AAGGAGGTTAGAGGTGTCTGCTGC \\
lnc-CMPK2-1:3 & TCAATAGAGAGGCAGACATACACA & ACAAGAAACACAGCACTAACAACA \\
lnc-GLI3-4:1 & GATGTGGTGGGTTCTCCAGTGTGA & TTTCCATCTTGCCTTCATTGTTTT \\
NR_104048 & AGTTTCCTTTTCATTGTTTTTTGC & GATCCTGTTTGCTACTGCCAGA \\
MEG3 & CTTTTCTGGGGGAATGGGG & AGAGGGGTGGGAAGGGACT \\
lnc-WRN-10:1 & ACATCAAGCTGTAACCAACCCAAC & TGCCTCTTCATCCACACTACCAAA \\
ENST00000583044 & AAATAACCCTATCAATCACCAAG & AGAGGAGAGAGATCAGGAAACC \\
ENST00000527317 & ACCAGAATGAGGTAAAAGAAGA & TGAGAGTGTGTGAGAACAAAG \\
GAPDH & ATCTTCCAGGAGCGAGATCCC & TGAGTCCTTCCACGATACCAA
\end{tabular}

lncRNA, long non-coding RNAs.
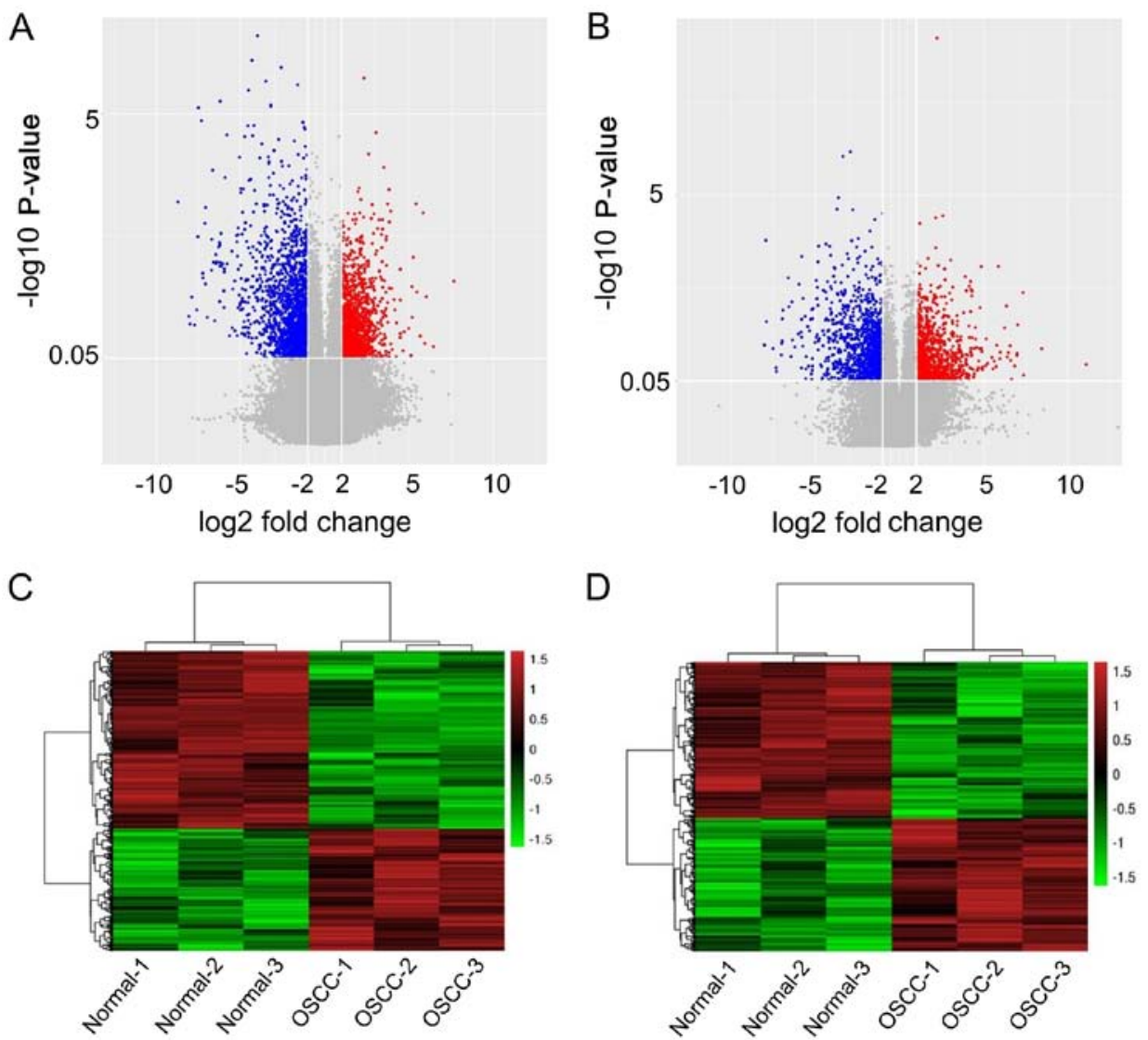

Figure 1. Volcano plot and hierarchical clustering analysis of differentially expressed (A and C) lncRNAs and (B and D) mRNAs between OSCC and paired adjacent normal tissues. The $\mathrm{x}$-axis and $\mathrm{y}$-axis in the volcano plot represent fold changes and P-values, respectively. The red dots to the right represent OSCC tissues, and the blue dots to the left represent paired adjacent normal tissues. For hierarchical clustering, black color represents 0 , indicating that expression of these genes was not altered. Red color indicates that the expression level was increased. Green color indicates that the expression level was decreased. The color intensity indicates the degree of gene upregulation or downregulation. lncRNAs, long non-coding RNAs; OSCC, oral squamous cell carcinoma.

transcription of total RNA was performed using PrimeScript RT reagent kit with gDNA Eraser (Takara Biotechnology Co., Ltd., Dalian, China). SYBR-Green qPCR Master Mix (Takara Biotechnology Co., Ltd.) was used for qRT-PCR assay according to the manufacturer's protocol. The thermocycling conditions were as follows: A denaturation step $10 \mathrm{~min}$ at $95^{\circ} \mathrm{C}$, followed by 40 cycles of $15 \mathrm{sec}$ at $95^{\circ} \mathrm{C}$ and $30 \mathrm{sec}$ at $60^{\circ} \mathrm{C}$ (adjusted with the Tm of different lncRNAs), $30 \mathrm{sec}$ at $72^{\circ} \mathrm{C}$. The housekeeping gene GAPDH was selected as an internal control. The $2^{-\Delta \Delta \mathrm{Cq}}$ method was used to measure relative expression levels, and each sample was analyzed at least in triplicate (16). Specific primers of each gene are listed in Table I. 
Table II. Relationship between the expression level of 4 critical node lncRNAs with the pathological characteristics of the OSCC patients $(\mathrm{N}=72)$.

\begin{tabular}{|c|c|c|c|c|c|c|c|c|c|}
\hline \multirow{2}{*}{$\begin{array}{l}\text { Clinical } \\
\text { characteristics }\end{array}$} & \multirow{2}{*}{$\begin{array}{l}N \\
72\end{array}$} & \multicolumn{2}{|c|}{ ENST00000583044 } & \multicolumn{2}{|c|}{ NR_104048 } & \multicolumn{2}{|c|}{ lnc-WRN-10:1 } & \multicolumn{2}{|c|}{ ENST00000527317 } \\
\hline & & High & Low & High & Low & High & Low & High & Low \\
\hline \multicolumn{10}{|l|}{ Age (years) } \\
\hline$<50$ & 32 & 14 & 18 & 15 & 17 & 14 & 18 & 15 & 17 \\
\hline$\geq 50$ & 40 & 16 & 24 & 13 & 27 & 12 & 28 & 15 & 25 \\
\hline P-value & & \multicolumn{2}{|c|}{0.748} & \multicolumn{2}{|c|}{0.214} & \multicolumn{2}{|c|}{0.227} & \multicolumn{2}{|c|}{0.423} \\
\hline \multicolumn{10}{|l|}{ Sex } \\
\hline Male & 35 & 16 & 19 & 16 & 19 & 15 & 20 & 16 & 19 \\
\hline Female & 37 & 14 & 23 & 12 & 25 & 11 & 26 & 14 & 23 \\
\hline P-value & & \multicolumn{2}{|c|}{0.498} & \multicolumn{2}{|c|}{0.248} & \multicolumn{2}{|c|}{0.246} & \multicolumn{2}{|c|}{0.498} \\
\hline \multicolumn{10}{|l|}{ Smoking } \\
\hline Yes & 42 & 15 & 27 & 16 & 26 & 14 & 28 & 18 & 24 \\
\hline No & 30 & 15 & 15 & 12 & 18 & 12 & 18 & 12 & 18 \\
\hline $\mathrm{P}$-value & & \multicolumn{2}{|c|}{0.225} & \multicolumn{2}{|c|}{0.870} & \multicolumn{2}{|c|}{0.561} & \multicolumn{2}{|c|}{0.808} \\
\hline \multicolumn{10}{|l|}{ Tumor location } \\
\hline Tongue cancer & 30 & 13 & 17 & 12 & 18 & 11 & 19 & 16 & 14 \\
\hline Gingival carcinoma & 22 & 7 & 15 & 8 & 14 & 7 & 15 & 5 & 17 \\
\hline Carcinoma of the buccal mucosa & 10 & 5 & 5 & 5 & 5 & 3 & 7 & 4 & 6 \\
\hline Others & 10 & 5 & 5 & 3 & 7 & 5 & 5 & 5 & 5 \\
\hline P-value & & \multicolumn{2}{|c|}{0.686} & \multicolumn{2}{|c|}{0.819} & \multicolumn{2}{|c|}{0.758} & \multicolumn{2}{|c|}{0.156} \\
\hline \multicolumn{10}{|l|}{ Clinical stage } \\
\hline $\mathrm{T} 1$ & 28 & 20 & 8 & 20 & 8 & 17 & 11 & 18 & 10 \\
\hline $\mathrm{T} 2$ & 25 & 4 & 21 & 3 & 22 & 5 & 20 & 6 & 19 \\
\hline $\mathrm{T} 3$ & 15 & 5 & 10 & 4 & 11 & 3 & 12 & 5 & 10 \\
\hline $\mathrm{T} 4$ & 4 & 1 & 3 & 1 & 3 & 1 & 3 & 1 & 3 \\
\hline P-value & & & & & 001 & & & & \\
\hline Lymphatic metastasis & & & & & & & & & \\
\hline Yes & 36 & 10 & 26 & 9 & 27 & 7 & 29 & 9 & 27 \\
\hline No & 36 & 20 & 16 & 19 & 17 & 19 & 17 & 21 & 15 \\
\hline $\mathrm{P}$-value & & & & & 16 & & & & \\
\hline Distant metastasis & & & & & & & & & \\
\hline Yes & 18 & 3 & 15 & 3 & 15 & 3 & 15 & 2 & 16 \\
\hline No & 54 & 27 & 27 & 25 & 29 & 23 & 31 & 28 & 26 \\
\hline P-value & & & & & 26 & & & & \\
\hline
\end{tabular}

lncRNA, long non-coding RNAs; OSCC, oral squamous cell carcinoma.

Statistical analysis. Statistical analysis was performed using SPSS statistical software package (version 22.0; IBM Corp., Armonk NY, USA). Data are expressed as the mean \pm standard deviation (SD). Student's t-test was used to compare different groups. The correlation between the lncRNA expression levels and the clinicopathological factors was analyzed using the Chi-square tests. Survival plots were generated by Kaplan-Meier analysis, and the log-rank test was used to assess the significance of the differences. P-values $<0.05$ were considered to indicate a statistically significant result.

\section{Results}

Patient information. The clinical characteristics of the 72 patients are summarized in Table II.

Volcano plot and hierarchical clustering analysis. In the volcano plot, after normalization and standardization, different signals were distributed in corresponding regions. Gray signals indicated that the detected genes did not meet the screening criteria. The results indicated that there were many lncRNAs and mRNAs that were 
differentially expressed between OSCC and normal tissues (Fig. 1A and B). Hierarchical clustering analysis determined correlations among samples through grouping at the gene level. In the hierarchical clustering analysis, each column represented one sample, and each row represented one gene. Hierarchical clustering analysis showed that the expression profiles were significantly different between OSCC and paired adjacent normal tissues (Fig. 1C and D).

Differentially expressed $\operatorname{lncR} N A s$ and $m R N A s$. To explore the role of IncRNAs in OSCC, we performed a genome-wide analysis of lncRNA and mRNA expression in OSCC and normal tissues. The results of the microarray assays showed that 2,294 differentially expressed lncRNAs (accounting for $2.9 \%$ of all detectable lncRNAs) and 1,938 differentially expressed mRNAs (accounting for 10.3\% of all detectable mRNAs) were identified. Furthermore, 933 lncRNAs and 891 mRNAs were upregulated and 1,361 lncRNAs and 1,047 mRNAs were downregulated. The most upregulated lncRNA and mRNA were MANSC4-8:1 $(\mathrm{FC}=201.36)$ and MMP7 ( $F C=2167.59)$, respectively. The most downregulated lncRNA and mRNA were NR_117092 $(\mathrm{FC}=418.62)$ and IL36A (FC=257.61), respectively. The top 20 dysregulated lncRNAs and mRNAs are summarized in Tables III and IV, respectively.

Construction of the lncRNA-mRNA co-expression network. Through construction of a co-expression network, we identified 306 differentially expressed lncRNAs interacting with other selected mRNAs and lncRNAs. According to the results, ENST00000583044, NR_104048, lnc-WRN-10:1 and ENST00000527317 were the four lncRNAs with the most frequent interactions (Fig. 2A and D). These four lncRNAs were node genes for the entire network, with relationship coefficients of 22, 22, 21 and 20. Other genes that did not directly interact with these four node genes interacted with them via indirect means through other relevant genes.

GO and KEGG pathway analyses. The differentially expressed mRNAs were processed for GO annotation. The results showed that the differentially expressed genes were enriched in molecular function (MF), biological process (BP) and cellular component (CC). Furthermore, three functions with the most enriched genes were interleukin-1 binding (GO:0019966; Ontology: molecular function; $\mathrm{P}=0.0003$ ), response to interferon- $\alpha$ (GO:0035455; Ontology: Biological process; $\mathrm{P}=1.37 \mathrm{E}-10)$ and FHF complex (GO:0070695; Ontology: Cellular component; $\mathrm{P}=0.0035$ ).

The results of the KEGG analysis demonstrated that differentially expressed mRNAs were mainly enriched in 38 biological pathways, including many cancer-related metabolic pathways, e.g., 'pathways in cancer' (enriched with 36 differentially expressed genes), 'bladder cancer' (enriched with 8 differentially expressed genes), 'metabolic pathways' (enriched with 109 differentially expressed genes), 'pancreatic cancer' (enriched with 11 differentially expressed genes) and 'PPAR signaling pathway' (enriched with 11 differentially expressed genes). The top 10 enriched GO and KEGG terms are summarized in the Tables V and VI.
A

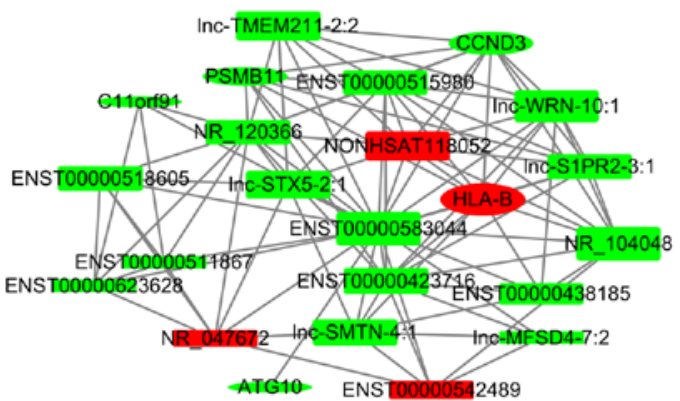

B

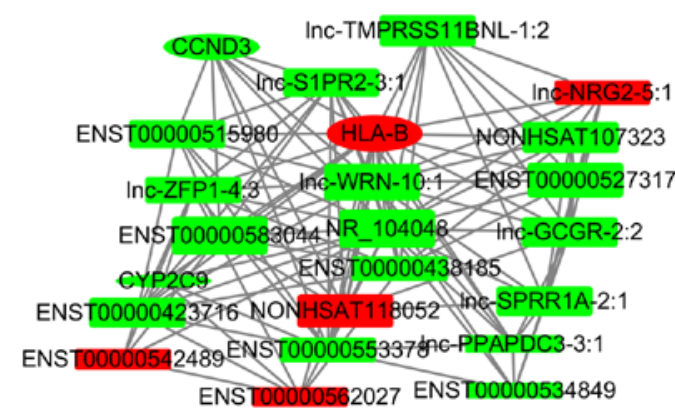

C

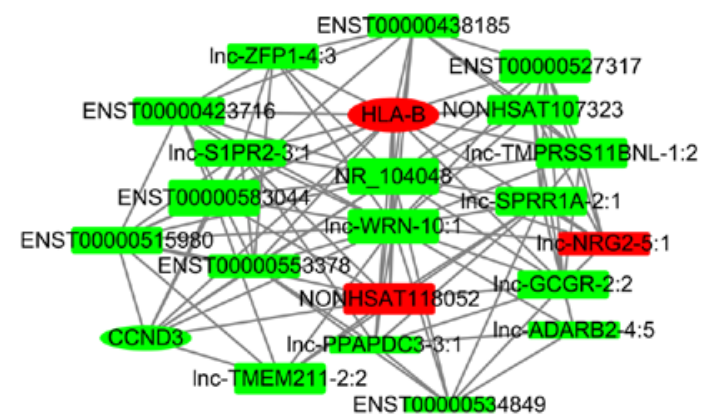

D

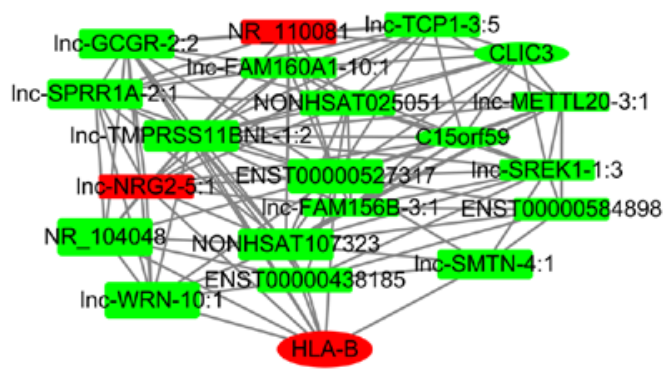

Figure 2. IncRNA-mRNA co-expression network. Genes directly interacting with (A) ENST00000583044, (B) NR_104048, (C) 1nc-WRN-10:1 and (D) ENST00000527317. Each point in the network represents a gene. The point size represents the relationship coefficient of each gene. The line represents the relationship between genes. The circles represent mRNAs and the boxes represent lncRNAs. Red color represents gene upregulation, and green color represents gene downregulation. lncRNAs, long non-coding RNAs; mRNA, messenger RNA.

Target gene prediction, GO analysis and KEGG pathway enrichment of IncRNAs. To explore the role of dysregulated lncRNAs in OSCC-related gene regulation and metabolic pathways, the target genes of lncRNAs were predicted using two independent algorithms. The results revealed that 1,470 dysregulated lncRNAs were identified to have cis or trans target genes, including 1,356 lncRNAs targeting 1,250 cis-genes, 370 lncRNAs targeting 2,454 trans-genes and 256 lncRNAs targeting both cis and 


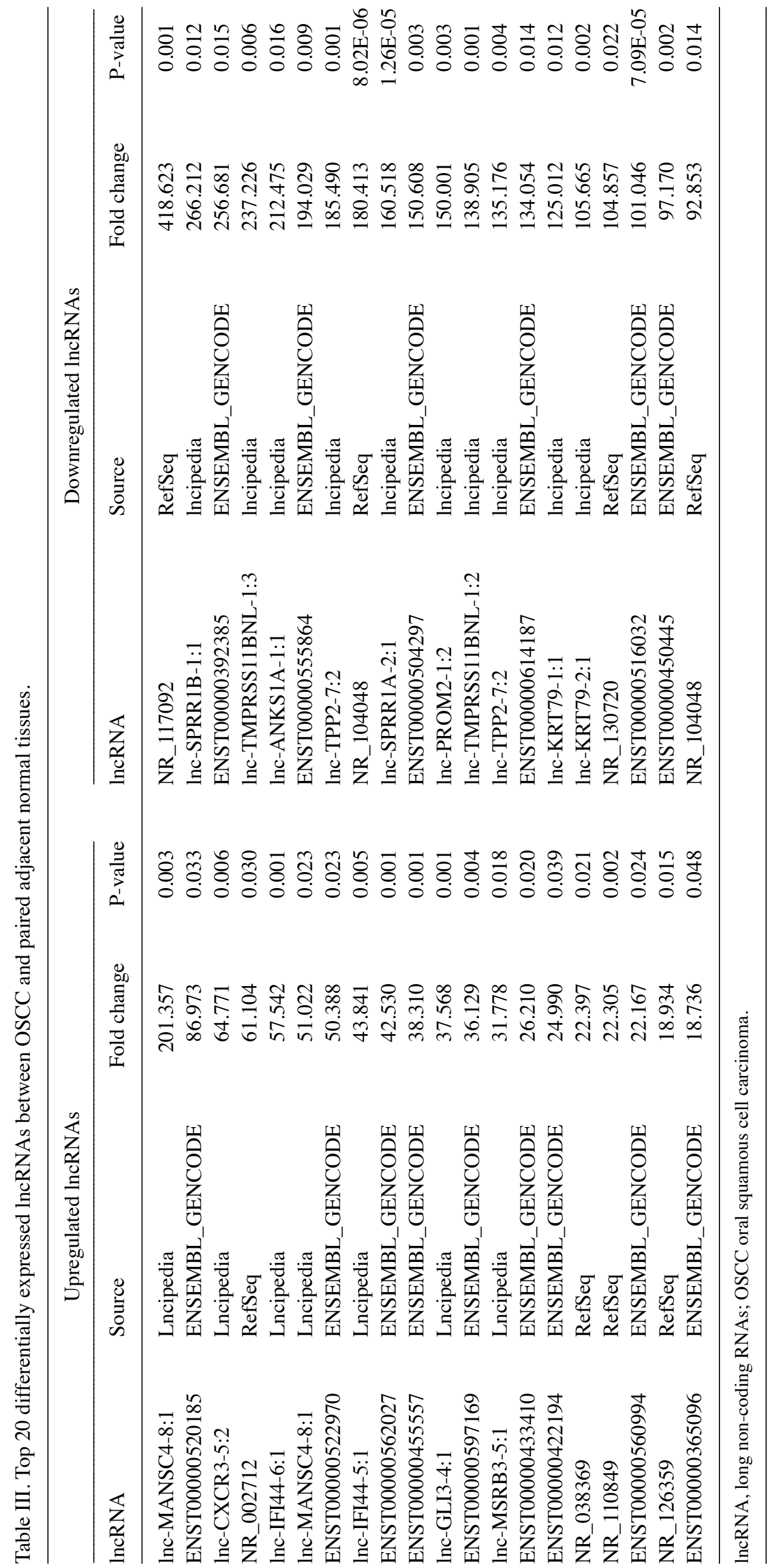


Table IV. Top 20 differentially expressed mRNAs between OSCC and paired adjacent normal tissues.

\begin{tabular}{|c|c|c|c|c|c|}
\hline \multicolumn{3}{|c|}{ Upregulated mRNAs } & \multicolumn{3}{|c|}{ Downregulated mRNAs } \\
\hline mRNA & Fold change & P-value & mRNA & Fold change & P-value \\
\hline MMP7 & 2167.591 & 0.024 & IL36A & 257.616 & 0.010 \\
\hline MMP10 & 344.744 & 0.012 & TMPRSS11B & 242.126 & $7.91 \mathrm{E}-05$ \\
\hline MMP7 & 163.871 & 0.038 & MAL & 237.820 & 0.003 \\
\hline MMP13 & 161.637 & 0.001 & CRNN & 180.673 & 0.011 \\
\hline PTHLH & 129.457 & 0.004 & SPINK7 & 159.889 & 0.027 \\
\hline CSAG3 & 125.283 & 0.014 & KRT4 & 157.082 & 0.033 \\
\hline CSAG3 & 124.324 & 0.015 & KRT13 & 129.559 & 0.015 \\
\hline SLCO1B3 & 114.167 & 0.025 & SPINK7 & 127.208 & 0.009 \\
\hline MMP12 & 99.936 & 0.046 & SPINK7 & 125.286 & 0.008 \\
\hline MMP1 & 97.071 & 0.020 & FAM3B & 124.222 & 0.003 \\
\hline IFIT2 & 94.992 & 0.010 & FAM3D & 120.592 & 0.002 \\
\hline HOXD11 & 87.675 & 0.014 & TMPRSS11A & 97.086 & 0.001 \\
\hline NLRP7 & 84.707 & 0.017 & KRT78 & 94.508 & 0.002 \\
\hline CXCL11 & 81.590 & 0.002 & KRT13 & 86.058 & 0.016 \\
\hline DNAH17 & 75.520 & 0.004 & ADH7 & 83.671 & 0.001 \\
\hline PTHLH & 63.483 & 0.013 & CLCA4 & 81.754 & 0.008 \\
\hline MMP1 & 62.481 & 0.018 & RHCG & 73.888 & 0.010 \\
\hline INHBA & 58.435 & 0.003 & SERPINB11 & 63.682 & 0.006 \\
\hline MMP11 & 54.858 & 0.009 & UPK1A & 62.443 & 0.001 \\
\hline MMP3 & 53.994 & 0.026 & ADH7 & 57.245 & 0.005 \\
\hline
\end{tabular}

mRNAs, messenger RNAs; OSCC, oral squamous cell carcinoma.

Table V. Top 10 of GO enrichment in different mRNAs.

\begin{tabular}{lcc}
\hline Description & Enrich factor & P-value \\
\hline Interleukin-1 binding & 9.676 & 0.002 \\
Response to interferon- $\alpha$ & 9.676 & $1.37 \mathrm{E}-10$ \\
Establishment of epithelial cell apical/basal polarity & 9.676 & 0.001 \\
Adenylate cyclase-inhibiting dopamine receptor signaling pathway & 8.708 & 0.003 \\
Regulation of synaptic vesicle priming & 8.708 & 0.003 \\
Oxidoreductase activity & 8.708 \\
PML body organization & 8.708 \\
Positive regulation of gonadotropin secretion & 8.708 \\
Negative regulation of viral-induced cytoplasmic pattern & 8.708 \\
recognition receptor signaling pathway & & 0.003 \\
Negative regulation of interferon- $\gamma$ biosynthetic process & 8.708 \\
\hline
\end{tabular}

GO, Gene Ontology; mRNA, messenger RNA.

trans target genes. We listed the top 30 terms of GO and KEGG enrichment using cis and trans methods (Figs. 3-6).

In the GO analysis, target genes with MF were mainly enriched in molecular binding, e.g., 'anion binding' and 'ion binding'. Target genes with CC functions were mainly enriched in cell organ and cell membrane, e.g., 'aggresome' and 'organelle membrane'. Target genes with the function of BP were mainly enriched in the synthesis process, e.g., 'cellular biosynthetic process' and 'organic substance biosynthetic processs' (Figs. 3 and 4). 
Table VI. Top 10 of pathway enrichment in different mRNAs.

\begin{tabular}{lcc}
\hline Description & Enrich factor & P-value \\
\hline Steroid biosynthesis & 5.383 & $4.60 \mathrm{E}-05$ \\
Glycosaminoglycan biosynthesis & 5.230 & $2.00 \mathrm{E}-05$ \\
Synthesis and degradation of ketone bodies & 4.262 & 0.028 \\
Biosynthesis of unsaturated fatty acids & 4.262 & 0.001 \\
Terpenoid backbone biosynthesis & 3.409 & 0.025 \\
Arginine and proline metabolism & 3.315 & $4.62 \mathrm{E}-05$ \\
$\alpha$-Linolenic acid metabolism & 3.196 & 0.017 \\
Thyroid cancer & 3.086 & 0.006 \\
ECM-receptor interaction & 2.858 & $2.14 \mathrm{E}-05$ \\
Tryptophan metabolism & 2.740 & 0.004
\end{tabular}

mRNA, messenger RNA.

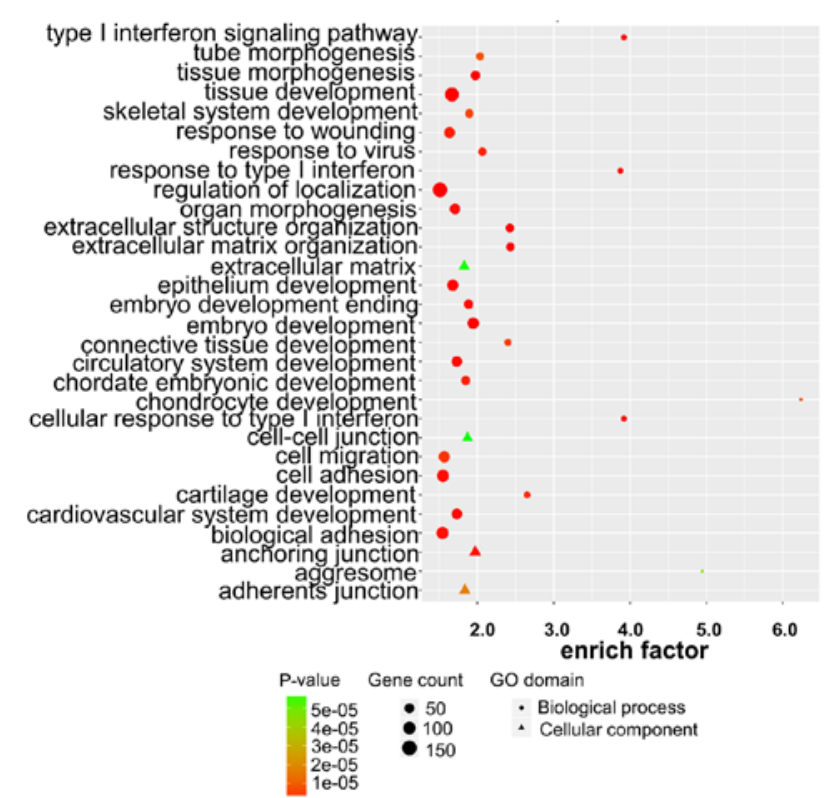

Figure 3. Top 30 GO enrichment terms using cis method. GO, Gene Ontology.

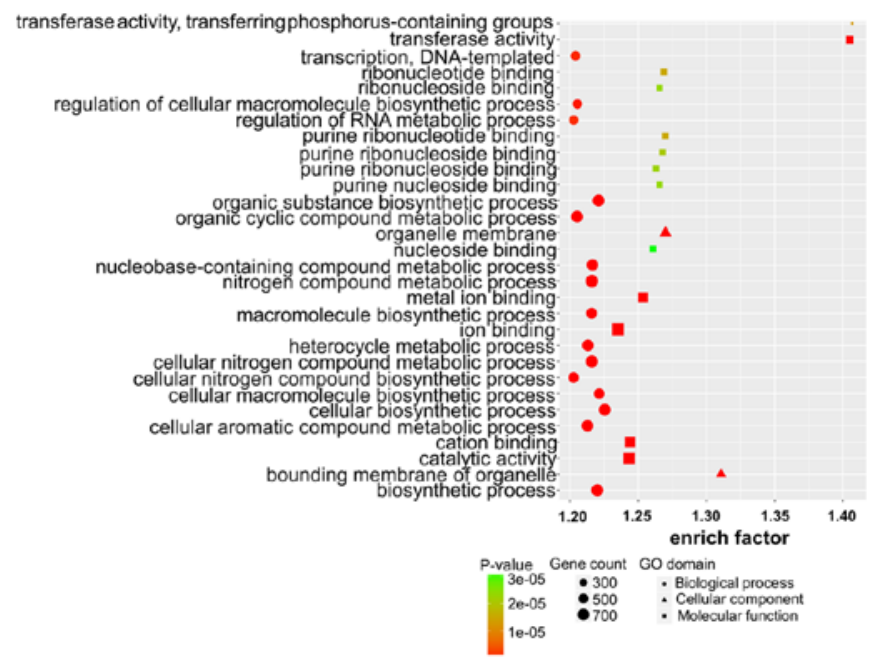

Figure 4. Top $30 \mathrm{GO}$ enrichment terms using trans method. GO, Gene Ontology.

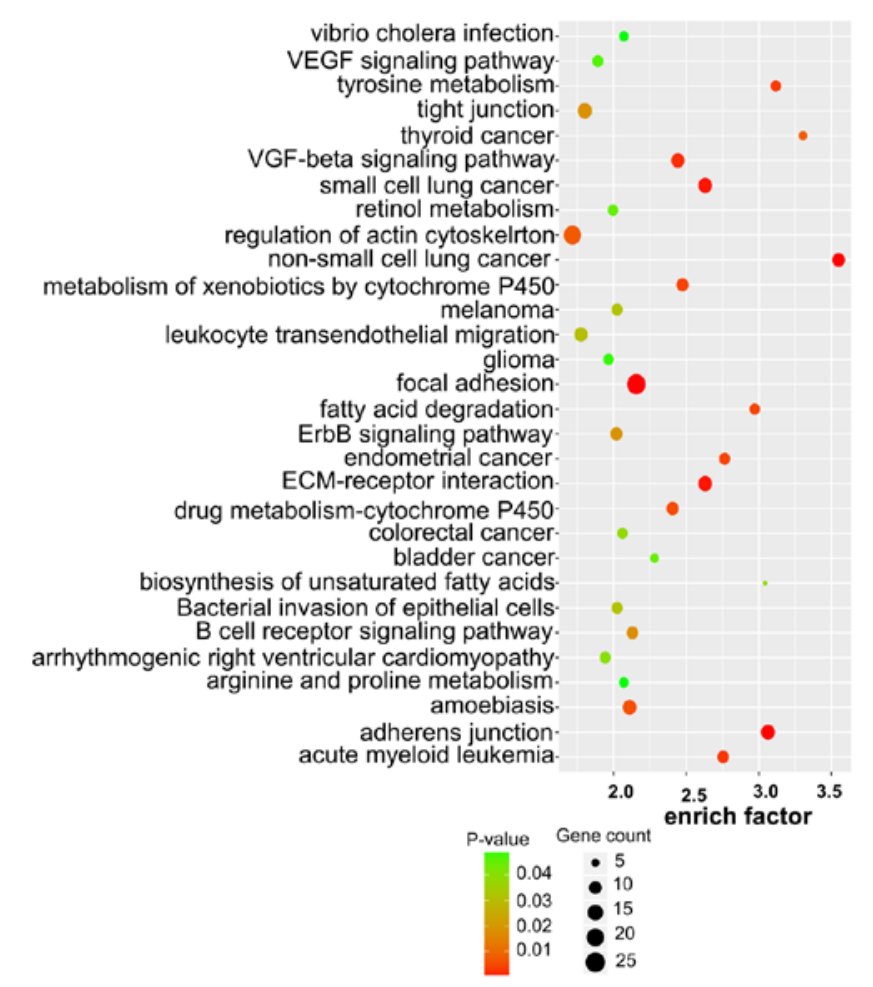

Figure 5. Top 30 KEGG enrichment terms using cis method. KEGG, Kyoto Encyclopedia of Genes and Genomes.

In the KEGG pathway enrichment, the target genes were enriched in 43 pathways. Among them, the cancer pathways were the main pathways. Target genes were also involved in other pathways, e.g., 'biosynthesis', 'metabolism' and 'signal pathway' (Figs. 5 and 6). These enriched metabolic pathways may be the key pathways involved in the regulation of tumorigenesis and development of OSCC by lncRNAs.

Validation by $q R T-P C R$. To verify the results of the microarray, 10 differentially expressed lncRNAs were randomly selected and assessed by qRT-PCR in 72 patient tissues. The data indicated that lnc-MANSC4-8:1, CXCR2P1, 


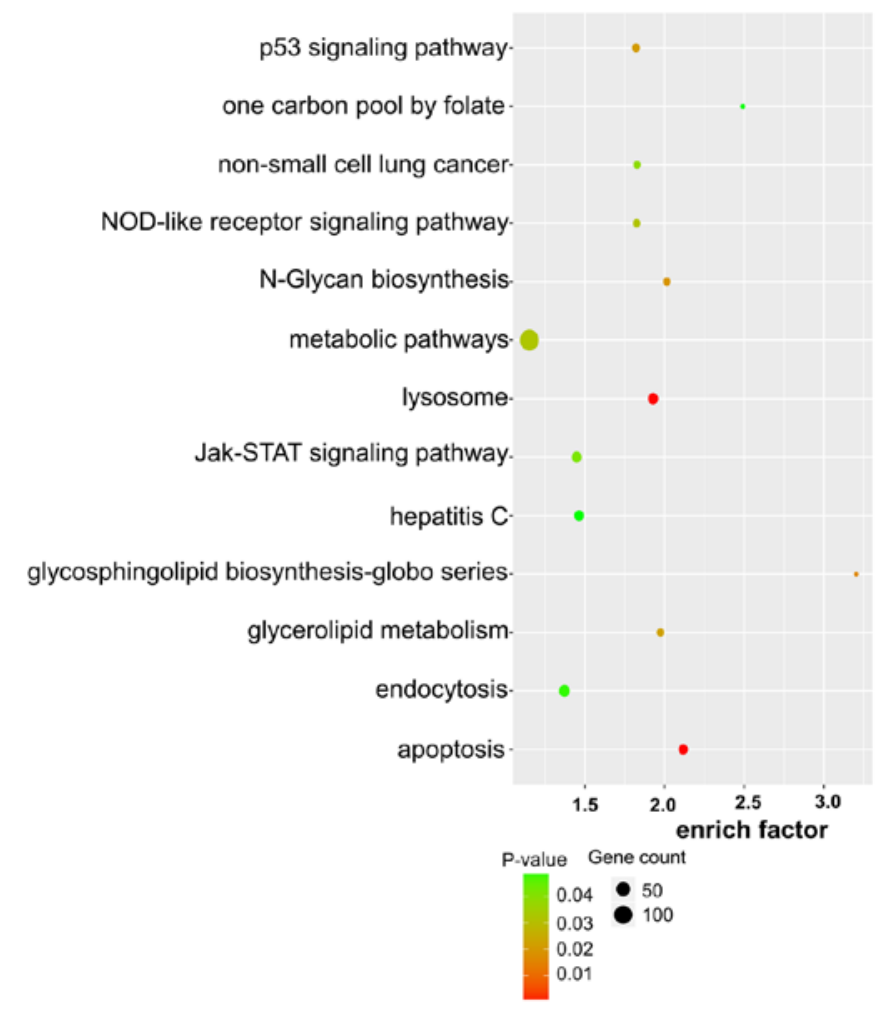

Figure 6. Top 30 KEGG enrichment terms using trans method. KEGG, Kyoto Encyclopedia of Genes and Genomes.

NRIR, lnc-CMPK2-1:3 and lnc-GLI3-4:1 were significantly upregulated, while TMPRSS11BNL, MEG3, lnc-WRN-10:1, DANCR and lnc-TPP2-7:2 were significantly downregulated in OSCC $(\mathrm{P}<0.05$, Fig. 7). The trend of dysregulated IncRNAs detected by qRT-PCR was consistent with those of the microarray assay.

Relationship between the expression of ENST00000583044, NR_104048, lnc-WRN-10:1, ENST00000527317 and the clinicopathologicalfeatures of OSCC patients. In 72 cases of OSCC, the expression levels of ENST00000583044, NR_104048, lnc-WRN-10:1 and ENST00000527317 were significantly lower than those in normal tissues $(\mathrm{P}<0.05)$ (Fig. 8). According to the average expression levels of ENST00000583044, NR_104048, Inc-WRN-10:1 and ENST00000527317 in OSCC, patients with OSCC were divided into a high ENST00000583044 expression group $(\geq 3.15)(n=30)$ and a low ENST00000583044 expression group $(<3.15)(n=42)$; a high NR_104048 expression group $(\geq 2.99)(\mathrm{n}=28)$ and a low NR_104048 expression group $(<2.99)(\mathrm{n}=44)$; a high lnc-WRN-10:1 expression group $(\geq 3.35)(\mathrm{n}=26)$ and a low lnc-WRN-10:1 expression group $(<3.35)(\mathrm{n}=46)$; and a high ENST00000527317 expression group $(\geq 2.91)(n=30)$ and a low ENST00000527317 expression group $(<2.91)(\mathrm{n}=42)$. We then analyzed the relationships among the expression of ENST00000583044, NR_104048, lnc-WRN-10:1, ENST00000527317 and age, sex, smoking status, tumor location, clinical stage, lymphatic metastasis, distant metastasis and survival status. The present study demonstrated that the expression of ENST00000583044,NR_104048,lnc-WRN-10:1 and ENST00000527317 was significantly correlated with
A

og 2 fold change $-10-8-6-4-2 \quad 0 \quad 2 \quad 4 \quad 6810$

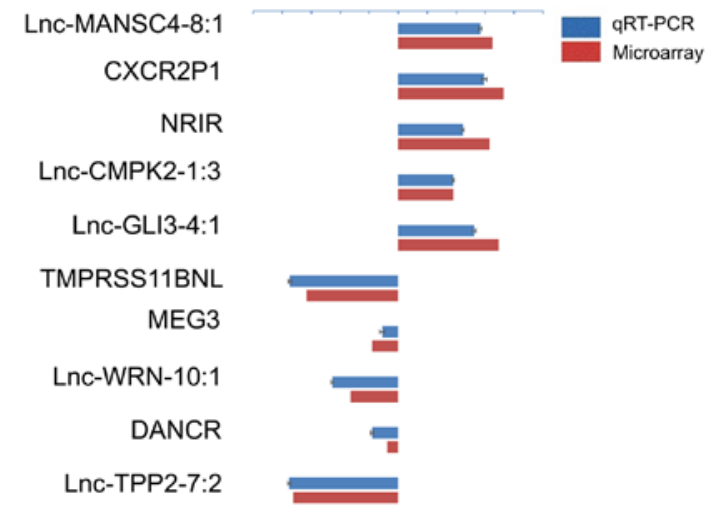

B

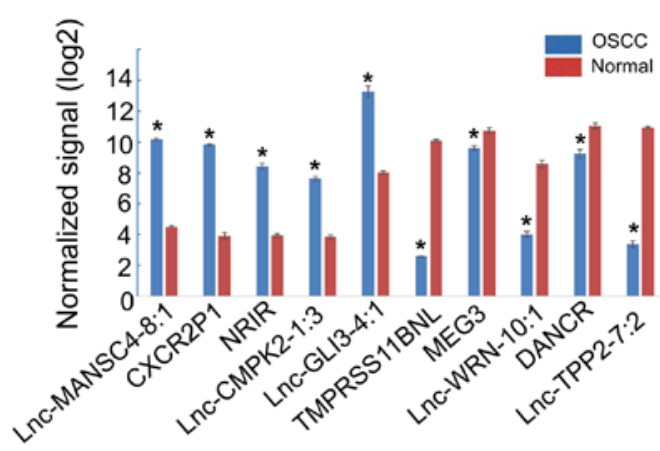

Figure 7. qRT-PCR validation of 10 differentially expressed lncRNAs (A) Comparison of fold change $[\log 2(\mathrm{O} / \mathrm{N})]$ of $\operatorname{lncRNAs}$ between the microarray and qRT-PCR results. (B) Normalized signal levels of lncRNAs in 72 pairs of OSCC and paired adjacent normal tissues. Data are presented as mean \pm standard deviation $(\mathrm{SD})$. IncRNAs, long non-coding RNAs; OSCC, oral squamous cell carcinoma. ${ }^{*} \mathrm{P}<0.05$.

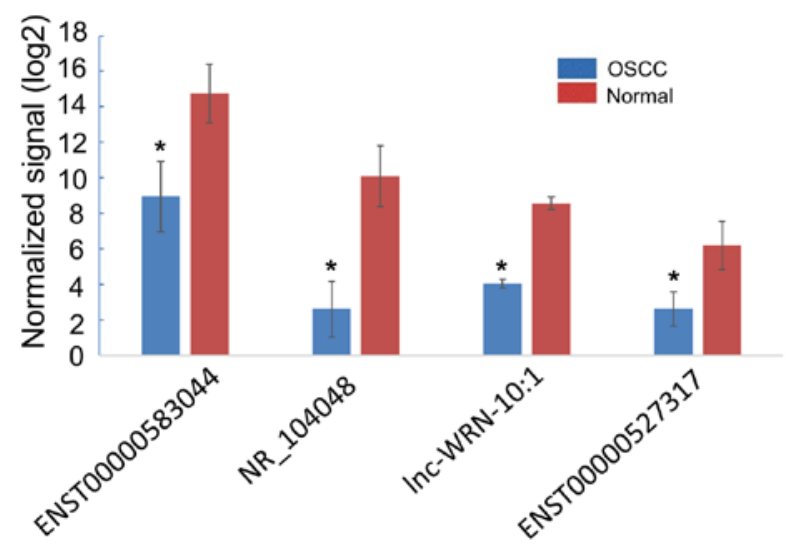

Figure 8. qRT-PCR validation of four critical node lncRNAs. Data are presented as mean \pm standard deviation (SD). lncRNAs, long non-coding RNAs. ${ }^{*} \mathrm{P}<0.05$.

clinical stage, lymphatic metastasis, distant metastasis and survival status $(\mathrm{P}<0.05)$. However, no significant associations were detected among the expression of ENST00000583044, NR_104048, lnc-WRN-10:1 and ENST00000527317 and age, sex, smoking and tumor location ( $\mathrm{P}>0.05$, Table II).

Kaplan-Meier analysis demonstrated that the median OS for patients with low expression of ENST00000583044, NR_104048, lnc-WRN-10:1 and ENST00000527317 was 

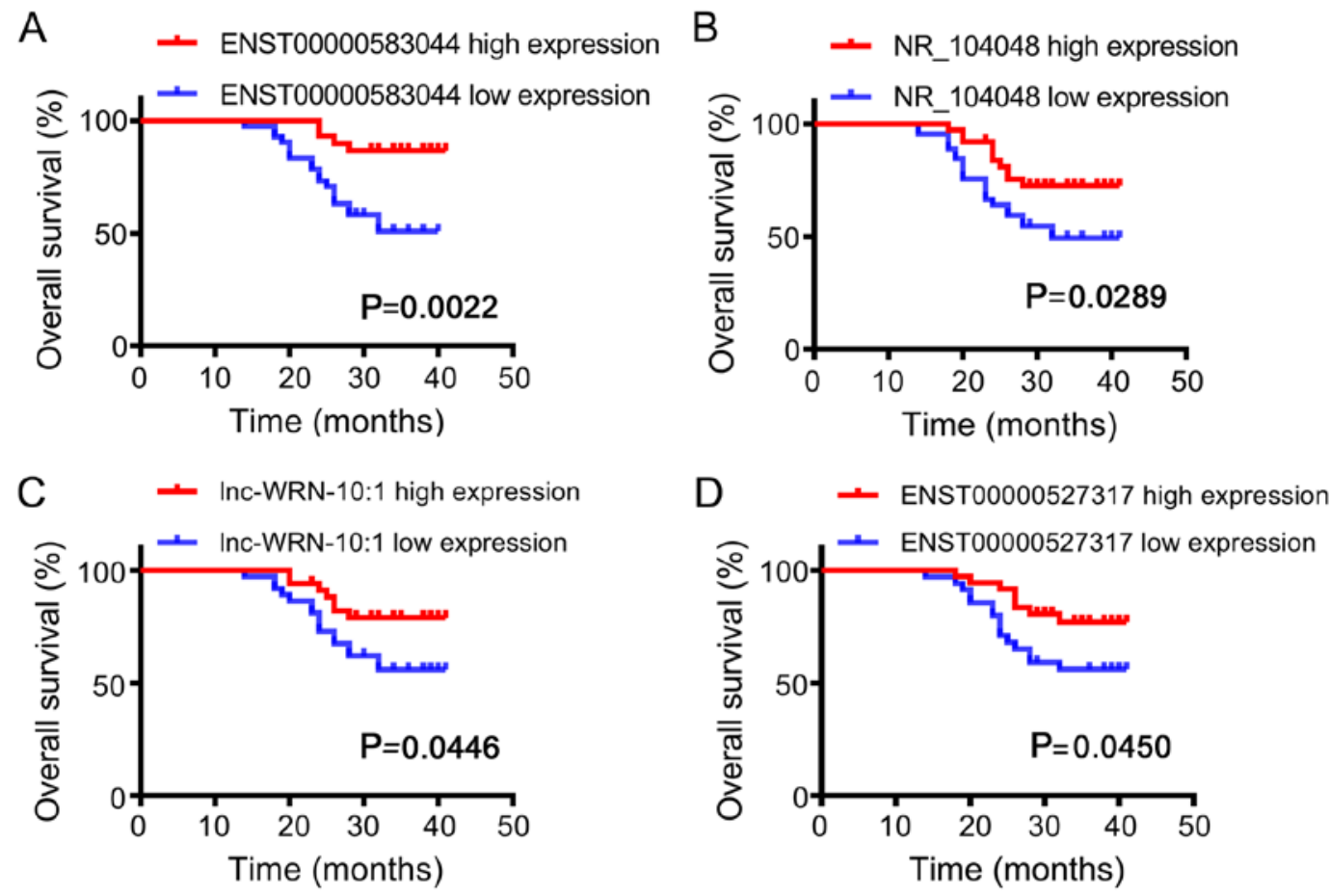

Figure 9. The relationship of four critical node lncRNAs expression with OS in OSCC patients. (A) The relationship of ENST00000583044 expression with OS in OSCC patients; (B) the relationship of NR_104048 expression with OS in OSCC patients; (C) the relationship of lnc-WRN-10:1 expression with OS in OSCC patients; (D) the relationship of ENST00000527317 expression with OS in OSCC patients. IncRNAs, long non-coding RNAs; OS, overall survival; OSCC, oral squamous cell carcinoma.
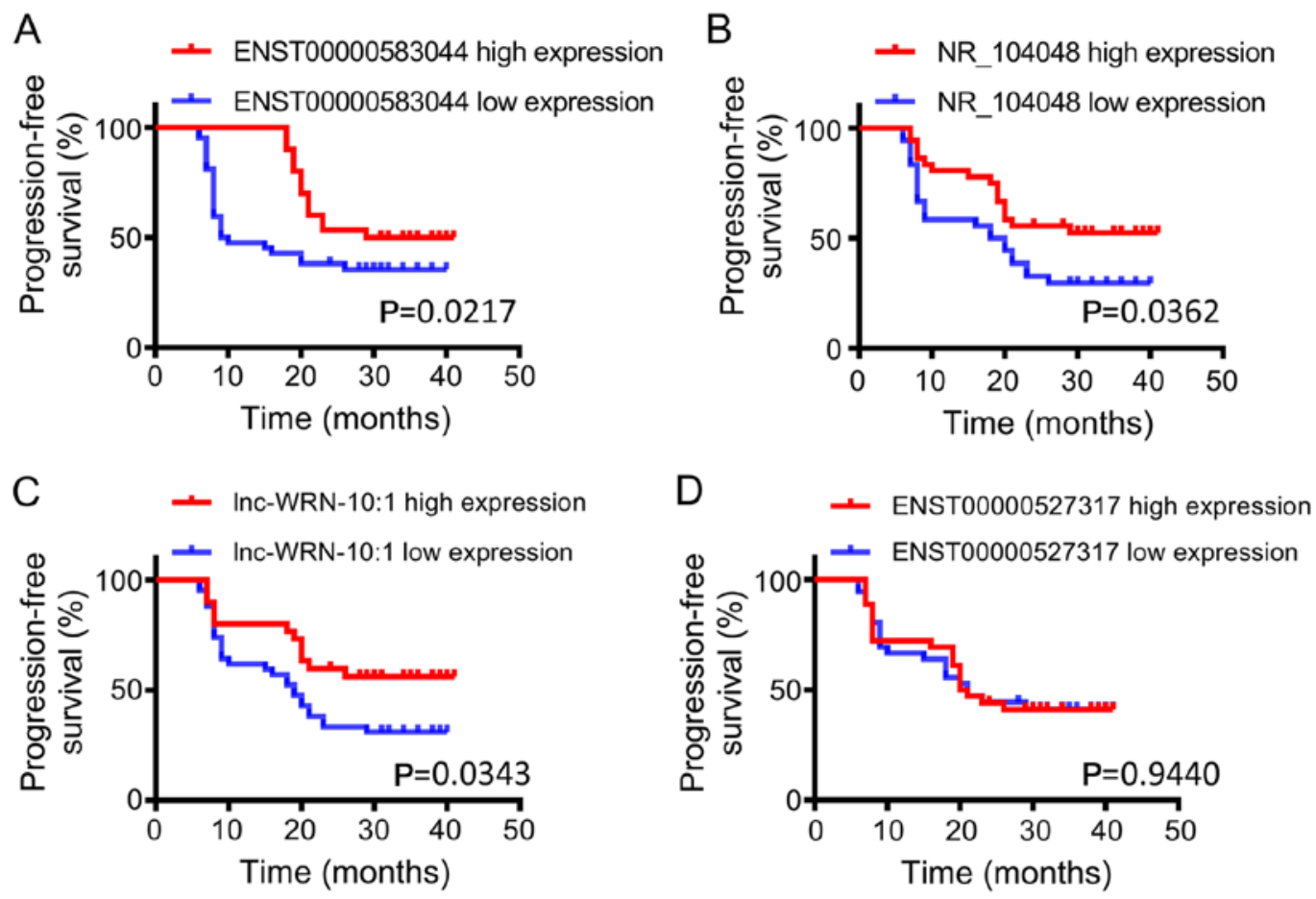

Figure 10. The relationship of four critical node lncRNAs expression with PFS in OSCC patients. (A) The relationship of ENST00000583044 expression with PFS in OSCC patients; (B) the relationship of NR_104048 expression with PFS in OSCC patients; (C) the relationship of lnc-WRN-10:1 expression with PFS in OSCC patients; (D) the relationship of ENST00000527317 expression with PFS in OSCC patients. IncRNAs, long non-coding RNAs; OSCC, oral squamous cell carcinoma; PFS, progression-free survival.

significantly lower than that in patients with high expression of these factors $(\mathrm{P}<0.05)$ (Fig. 9). Furthermore, the median PFS for patients with high expression of
ENST00000583044, NR_104048 and lnc-WRN-10:1 was significantly higher than that in patients with low expression $(\mathrm{P}<0.05)$ (Fig. 10). 


\section{Discussion}

Tumorigenesis and development of oral squamous cell carcinoma (OSCC) consists of a complex process, and the underlying mechanisms remain poorly understood. The aim of the present study was to explore the relationship between lncRNAs and OSCC. IncRNAs were initially considered 'noise' without any biological functions in the human genome. They are now known to play important roles in gene expression, and their differential expression may affect corresponding functional performance (4). Subsequently, many studies have demonstrated that lncRNAs are involved in many important regulatory processes, including $\mathrm{X}$-chromosome silencing, genomic imprinting, chromatin modification, transcriptional activation, transcriptional interference and intranuclear transport $(17,18)$. In the present study, we used an expression profile microarray to identify differentially expressed genes in OSCC at the whole genome level. The results revealed a large number of differentially expressed lncRNAs and mRNAs, some of which may be important genes involved in tumorigenesis and the development of OSCC. For example, MALAT1 promotes the invasion and metastasis of lung cancer (19). MEG3 expression levels were highly correlated with invasion and metastasis of gastric cancer $(20,21)$. GAS5 indicates a poor prognosis in ovarian cancer (22). Feng et al showed that lncRNAs were abnormally expressed in OSCC and metastatic tissue samples (5). Recent studies have shown that HOTAIR is highly expressed in OSCC and is associated with the biological behavior of tumor invasion and metastasis $(6,23)$.

In view of the complex transcriptional regulatory mechanisms of lncRNAs and their ability to form a variety of secondary functional structures, their biological functions cannot be predicted based solely on nucleic acid sequence $(24,25)$. IncRNA loci are often located in intronic regions of the coding gene and thus may affect expression of its adjacent genes (26-28). To investigate the relationship between lncRNAs and mRNAs, coexpression networks of dysregulated lncRNAs and mRNAs were constructed based on differential expression. Differentially expressed genes were assigned into subnetworks associated with phenotypic functions to roughly deduce the function of lncRNAs in this subnetwork and predict possible regulatory mechanisms. We identified 4 lncRNAs with high correlations with other genes. ENST00000583044, NR_104048, Inc-WRN-10:1 and ENST00000527317, which were found in the present study, belonged to downregulated IncRNAs and have never previously been reported in OSCC or any other solid tumors. Our data are the first to reveal these four critical node genes. We speculate that these results may depend on tumor heterogeneity. As with any cancer, OSCC is also highly heterogeneous and is characterized by different genetic backgrounds, different pathological types, different differentiation states, different gene mutation and transcriptional patterns and proteome expression profiles $(29,30)$. We further examined the expression of these 4 genes in 72 patients with OSCC and normal tissues and analyzed their relationship with the clinicopathological features and prognosis. We found that these 4 genes were downregulated in OSCC. Moreover, their expression was not correlated with age, sex, smoking, or tumor location but was related to clinical stage, lymphatic metastasis, distant metastasis and survival status. Furthermore, low expression levels of these 4 lncRNAs contributed to poor median PFS and OS.
In addition, by constructing a co-expression network of differentially expressed genes, we found that some lncRNAs, e.g., NR_002812, regulate many genes, including SP100 and B2M. Previous studies have shown that the SP100 protein is involved in viral infection, virus-related protein interaction and self-ubiquitination regulation, and plays an important role in interferon and p53 signaling pathways $(31,32)$. p53 protein inhibits the growth and invasion of oral malignancy by regulating the phosphorylation of AKT $(25,33,34)$. Although the specific mechanism and related signaling pathways need to be further studied, they can be used as molecular markers for early diagnosis, treatment and prognostic evaluation of OSCC.

Since lncRNAs do not encode protein, we studied the mechanism of pathogenesis from another angle by enriching the biological function of differentially expressed mRNAs. In the present study, GO and KEGG pathway analyses were performed to examine the biological function of dysregulated genes $(35,36)$. GO functional annotations indicated that the differentially expressed genes were enriched in different BP, $\mathrm{CC}$ and MF categories. KEGG enrichment identified many metabolic pathways associated with cancer, e.g., 'metabolic pathways' and 'pathways in cancer', demonstrating that OSCC is associated with cell structure changes, metabolic process disorders, tumor suppressor genes and oncogene signaling pathway abnormalities and was a consequence of multiple intracellular and external factors. These results further verified the results of the microarray assay. IncRNAs can guide gene expression in either a cis or trans manner. In the present study, two independent algorithms were used to predict $c i s$ and trans target genes of differentially expressed IncRNAs in OSCC. Through GO functional annotation and KEGG pathway analysis, we found that these target genes regulate relevant OSCC proteins and affect OSCC tumorigenesis and development via their functions in organ, molecular binding, metabolism and cancer pathways. Target prediction of IncRNAs provides important information for further study of potential functional lncRNAs and target genes in OSCC.

The present study also has several limitations including its small sample size. In the future, our results need to be validated in large-scale samples.

In conclusion, in the present study, 2,294 dysregulated lncRNAs and 1,938 dysregulated mRNAs were identified by a microarray assay. We explored 4 critical $\operatorname{lncRNAs}$ nodes, which may play an important role in the pathogenesis of OSCC. GO and pathway analyses indicated that the functions and enriched pathways of many dysregulated genes were associated with cancer. The potential target genes of dysregulated lncRNAs were enriched in 43 KEGG pathways, and cancer pathways were the primary enrichment pathways. These results provide new insight into the molecular markers and therapeutic targets for OSCC.

\section{Acknowledgements}

Not applicable.

\section{Funding}

The present study was supported by the National Natural Science Foundation of China (no. 81372150). 


\section{Availability of data and materials}

The datasets used during the present study are available from the corresponding author upon reasonable request.

\section{Authors' contributions}

YLQ, YHL and BHL acquired the data and created a draft of the manuscript; JDB, WJW, MH and PK conducted the experiments and collected the data; YLQ and YHL interpreted the data, performed the statistical analysis and analyzed the results; BHL revised and approved the final version of the manuscript. All authors read and approved the manuscript and agree to be accountable for all aspects of the research in ensuring that the accuracy or integrity of any part of the work are appropriately investigated and resolved.

\section{Ethics approval and consent to participate}

The present study was approved by the Ethics Committee of the Fourth Affiliated Hospital of Hebei Medical University (Shijiazhuang, China) and written informed consent was obtained from all subjects.

\section{Patient consent for publication}

Not applicable.

\section{Competing interests}

The authors declare that they have no competing interests.

\section{References}

1. Poveda-Roda R, Bagán JV, Jiménez-Soriano Y, MargaixMuñoz M and Sarrión-Pérez G: Changes in smoking habit among patients with a history of oral squamous cell carcinoma (OSCC). Med Oral Patol Oral Cir Bucal 15: e721-e726, 2010.

2. Messadi DV: Diagnostic aids for detection of oral precancerous conditions. Int J Oral Sci 5: 59-65, 2013.

3. Cui Z, Ren S, Lu J, Wang F, Xu W, Sun Y, Wei M, Chen J, Gao X, $\mathrm{Xu} \mathrm{C}$, et al: The prostate cancer-up-regulated long noncoding RNA PlncRNA-1 modulates apoptosis and proliferation through reciprocal regulation of androgen receptor. Urol Oncol 31: 1117-1123, 2013.

4. Zhou X, Liu S, Cai G, Kong L, Zhang T, Ren Y, Wu Y, Mei M, Zhang $L$ and Wang $X$ : Long non coding RNA MALAT1 promotes tumor growth and metastasis by inducing epithelial-mesenchymal transition in oral squamous cell carcinoma. Sci Rep 5: 15972, 2015.

5. Feng L, Houck JR, Lohavanichbutr P and Chen C: Transcriptome analysis reveals differentially expressed lncRNAs between oral squamous cell carcinoma and healthy oral mucosa. Oncotarget 8: 31521-31531, 2017.

6. Wu Y, Zhang L, Zhang L, Wang Y, Li H, Ren X, Wei F, Yu W, Liu T, Wang X, et al: Long non-coding RNA HOTAIR promotes tumor cell invasion and metastasis by recruiting EZH2 and repressing E-cadherin in oral squamous cell carcinoma. Int J Oncol 46: 2586-2594, 2015.

7. Gupta RA, Shah N, Wang KC, Kim J, Horlings HM, Wong DJ, Tsai MC, Hung T, Argani P, Rinn JL, et al: Long non-coding RNA HOTAIR reprograms chromatin state to promote cancer metastasis. Nature 464: 1071-1076, 2010.

8. Eades G, Zhang YS, Li QL, Xia JX, Yao Y and Zhou Q: Long non-coding RNAs in stem cells and cancer. World J Clin Oncol 5: $134-141,2014$.
9. Zhang $\mathrm{L}, \mathrm{Xu} \mathrm{HG}$ and Lu C: A novel long non-coding RNA T-ALL-R-LncR1 knockdown and Par-4 cooperate to induce cellular apoptosis in T-cell acute lymphoblastic leukemia cells. Leuk Lymphoma 55: 1373-1382, 2014.

10. Zang W, Wang T, Huang J, Li M, Wang Y, Du Y, Chen X and Zhao G: Long noncoding RNA PEG10 regulates proliferation and invasion of esophageal cancer cells. Cancer Gene Ther 22: 138-144, 2015.

11. Wang Y, He L, Du Y, Zhu P, Huang G, Luo J, Yan X, Ye B, $\mathrm{Li} \mathrm{C}, \mathrm{Xia} \mathrm{P}$, et al: The long noncoding RNA lncTCF7 promotes self-renewal of human liver cancer stem cells through activation of Wnt signaling. Cell Stem Cell 16: 413-425, 2015.

12. Loewen G, Jayawickramarajah J, Zhuo Y and Shan B: Functions of lncRNA HOTAIR in lung cancer. J Hematol Oncol 7: 90, 2014.

13. Shi SJ, Wang LJ, Yu B, Li YH, Jin Y and Bai XZ: LncRNA-ATB promotes trastuzumab resistance and invasion-metastasis cascade in breast cancer. Oncotarget 6: 11652-11663, 2015.

14. Yan P, Luo S, Lu JY and Shen X: Cis- and trans-acting lncRNAs in pluripotency and reprogramming. Curr Opin Genet Dev 46: 170-178, 2017.

15. Tafer H and Hofacker IL: RNAplex: A fast tool for RNA-RNA interaction search. Bioinformatics 24: 2657-2663, 2008.

16. Livak KJ and Schmittgen TD: Analysis of relative gene expression data using real-time quantitative PCR and the $2^{-\Delta \Delta C_{\mathrm{T}}}$ method. Methods 25: 402-408, 2001.

17. Zhu DX, Zhu W, Fang C, Fan L, Zou ZJ, Wang YH, Liu P, Hong M, Miao KR, Liu P, et al: $\mathrm{miR}-181 \mathrm{a} / \mathrm{b}$ significantly enhances drug sensitivity in chronic lymphocytic leukemia cells via targeting multiple anti-apoptosis genes. Carcinogenesis 33: 1294-1301, 2012.

18. Kornienko AE, Guenzl PM, Barlow DP and Pauler FM: Gene regulation by the act of long non-coding RNA transcription. BMC Biol 11: 59, 2013.

19. Gutschner T, Hämmerle M, Eissmann M, Hsu J, Kim Y, Hung G, Revenko A, Arun G, Stentrup M, Gross M, et al: The noncoding RNA MALAT1 is a critical regulator of the metastasis phenotype of lung cancer cells. Cancer Res 73: 1180-1189, 2013.

20. Zhou Y, Zhang X and Klibanski A: MEG3 noncoding RNA: A tumor suppressor. J Mol Endocrinol 48: R45-R53, 2012.

21. Zhou Y, Zhong Y, Wang Y, Zhang X, Batista DL, Gejman R, Ansell PJ, Zhao J, Weng C and Klibanski A: Activation of p53 by MEG3 non-coding RNA. J Biol Chem 282: 24731-24742, 2007.

22. Ma N, Li S, Zhang Q, Wang H, Qin H and Wang S: Long non-coding RNA GAS5 inhibits ovarian cancer cell proliferation via the control of microRNA-21 and SPRY2 expression. Exp Ther Med 16: 73-82, 2018.

23. Lu MY, Liao YW, Chen PY, Hsieh PL, Fang CY, Wu CY, Yen ML, Peng BY, Wang DP, Cheng HC, et al: Targeting LncRNA HOTAIR suppresses cancer stemness and metastasis in oral carcinomas stem cells through modulation of EMT. Oncotarget 8: 98542-98552, 2017.

24. Zhang B, Arun G, Mao YS, Lazar Z, Hung G, Bhattacharjee G, Xiao X, Booth CJ, Wu J, Zhang C, et al: The lncRNA Malat1 is dispensable for mouse development but its transcription plays a cis-regulatory role in the adult. Cell Rep 2: 111-123, 2012.

25. Wang G, Li Z, Zhao Q, Zhu Y, Zhao C, Li X, Ma Z, Li X and Zhang Y: LincRNA-p21 enhances the sensitivity of radiotherapy for human colorectal cancer by targeting the Wnt/beta-catenin signaling pathway. Oncol Rep 31: 1839-1845, 2014.

26. Roy R, Singh R, Chattopadhyay E, Ray A, Sarkar N, Aich R, Paul RR, Pal M and Roy B: MicroRNA and target gene expression based clustering of oral cancer, precancer and normal tissues. Gene 593: 58-63, 2016.

27. Zaid KW, Nhar BM, Ghadeer Alanazi SM, Murad R, Domani A and Alhafi AJ: Lack of effects of recombinant human bone morphogenetic protein 2 on angiogenesis in oral squamous cell carcinoma induced in the syrian hamster cheek Pouch. Asian Pac J Cancer Prev 17: 3527-3531, 2016.

28. Zhang X, Gejman R, Mahta A, Zhong Y, Rice KA, Zhou Y, Cheunsuchon P, Louis DN and Klibanski A: Maternally expressed gene 3, an imprinted noncoding RNA gene, is associated with meningioma pathogenesis and progression. Cancer Res 70: 2350-2358, 2010.

29. Sasahira T and Kirita T: Hallmarks of cancer-related newly prognostic factors of oral squamous cell carcinoma. Int J Mol Sci 19: E2413, 2018.

30. Lala M, Chirovsky D, Cheng JD and Mayawala K: Clinical outcomes with therapies for previously treated recurrent/metastatic head-and-neck squamous cell carcinoma $(\mathrm{R} / \mathrm{M}$ HNSCC): A systematic literature review. Oral Oncol 84: 108-120, 2018. 
31. Wurdak M, Schneider M, Iftner T and Stubenrauch F: The contribution of SP100 to cottontail rabbit papillomavirus transcription and replication. J Gen Virol: Jan 24, 2018 (Epub ahead of print). doi: 10.1099/jgv.0.001012.

32. Berscheminski J, Brun J, Speiseder T, Wimmer P, Ip WH, Terzic M, Dobner T and Schreiner S: Schreiner, Sp100A is a tumor suppressor that activates p53-dependent transcription and counteracts E1A/E1B-55K-mediated transformation. Oncogene 35: 3178-3189, 2016

33. Kim T, Veronese A, Pichiorri F, Lee TJ, Jeon YJ, Volinia S, Pineau P, Marchio A, Palatini J, Suh SS, et al: p53 regulates epithelial-mesenchymal transition through microRNAs targeting ZEB1 and ZEB2. J Exp Med 208: 875-883, 2011.

34. Tan BS, Tiong KH, Choo HL, Chung FF, Hii LW, Tan SH, Yap IK, Pani S, Khor NT, Wong SF, et al: Mutant p53-R273H mediates cancer cell survival and anoikis resistance through AKT-dependent suppression of BCL2-modifying factor (BMF). Cell Death Dis 6: e1826, 2015.
35. Peng W, Gao W and Feng J: Long noncoding RNA HULC is a novel biomarker of poor prognosis in patients with pancreatic cancer. Med Oncol 31: 346, 2014.

36. Zhao Z, Bai J, Wu A, Wang Y, Zhang J, Wang Z, Li Y, Xu J and Li X: Co-LncRNA: Investigating the lncRNA combinatorial effects in GO annotations and KEGG pathways based on human RNA-Seq data. Database 2015: bav082, 2015.

(i) $\odot$ This work is licensed under a Creative Common Attribution-NonCommercial-NoDerivatives 4.0 International (CC BY-NC-ND 4.0) License. 